\title{
Plants used as painkiller in folk medicine in Turkey - III: Rheumatic pain
}

\author{
Meryem Şeyda Erbay *, Sezin Anıl, Gülay Melikoğlu \\ İstanbul University, Faculty of Pharmacy, Department of Pharmacognosy, Beyazit 34116 İstanbul, Turkey. \\ * Correspondence: mseydaerbay@gmail.com (M.Ş.E.); Tel: +90 212440 00 00/13594; ORCID No: 0000-0001-9889- \\ 9250.
}

Submitted: 28 March 2017 / Revised: 20 July 2017 / Accepted: 2 August 2017

\begin{abstract}
There are many plants used by the public in the treatment of various diseases in Turkey.The folk remedies prepared with these plants from which treatment and how they used have been reached to day-to-day by transferring the generations. The traditional treatment methods are recorded by ethnobotanical researches and it is aimed to contribute to drug development studies. In this study, which was prepared by screening of ethnobotanical researches, 72 taxa which used in traditional treatment against rheumatic pain in Turkey are reached and the scientific and local names, families, used parts of these taxa and the use forms in rheumatic pain were compiled. According to the research done, families that are commonly used for rheumatic pain are Ranunculaceae ( 8 taxa), Asteraceae ( 5 taxa), Brassicaceae (5 taxa), Lamiaceae (5 taxa), Apiaceae (4 taxa) and Liliaceae (4 taxa). The plants used for rheumatic pain contain analgesic, anti-inflammatory, antiseptic and rubefian compounds. Rubefian compound containing species are applied externally to the rheumatoid region and act by increasing the blood supply of the region. Analgesic, anti-inflammatory and antiseptic active species are used in the form of decoction / infusion both internally and externally.
\end{abstract}

KEYWORDS: Rheumatic pain; Traditional treatment; Medicinal plants; Turkey.

\section{INTRODUCTION}

Pain is an important health problem that affects millions of people every year and develops due to various causes and has a negative impact on quality of life. International health care organizations have defined pain as a sign of disease [1]. It is also accepted that pain is a disease in itself [2].

Turkey has a great diversity in terms of climate and geographical conditions. This diversity brings the advantage of having a rich plant cover. Approximately eleven thousand plant species are growing in our country, and three thousand of them are endemic [3-5]. From the early ages, the plants are used by people as food or to prevent health problems. Medicinal plants, which have been traditionally used by the public at the time, are recorded with ethnobotanical investigations. Such studies are a very important source of information for drug research. Folk medicine has an important place both in the world and in our country in health field. Traditional treatment is frequently used in the treatment of diseases, especially in less developed regions. In this study, plants which have traditionally been used as painkillers in Turkey have been identified by screening ethnobotanical studies. The findings are classified according to pain types and will be published as an article series. This study, which is third article of series, contains the plants used against rheumatic pain.

Diseases that cause swelling, pain, restriction of movement, and various disorders in the internal organs are called rheumatism. Factors such as genetic, age, gender, some medications, accidental injuries, climate, some diseases affect the pattern and severity of the disease. Rheumatism is divided into two, as inflammatory and non-inflammatory. Inflammatory rheumatism is the rheumatism caused by microbes that break down the immune system. In non-inflamed rheumatism, thinning and erosion occurs in the joints. Bone peel may occur. It can be caused by an accident.

Rheumatic diseases can be divided into 4 groups depending on where they are:

- Soft tissue rheumatism (the most important and most common type.)

- Rheumatoid arthritis

- Internal organ rheumatism

- Multiple facts [6].

How to cite this article: Erbay MŞ, Anıl S, Melikoğlu G. Plants used as painkiller in folk medicine in Turkey - III: Rheumatic pain. Marmara Pharm J 2018;22(2):133-141. 
Rheumatic symptoms are distinguished by the following seven characteristics: 1. pain or discomfort, usually perceived in the vicinity of one or more joints (including the spine); 2 . pain on motion of the affected area(s); 3. soreness (to the touch) of the affected region(s); 4. stiffness of the affected part(s), especially after a period of immobility; 5 . symptomatic improvement after mild exercise, but worsening after vigorous exercise; 6. symptomatic worsening in response to climatic factors, especially falling barometric pressure and rising humidity; 7. symptomatic improvement in response to warming the affected area(s). Not all rheumatic pain syndromes have all seven characteristics, but most will at least have the first four. Rheumatic pain is almost always localized. It may be localized to one region of the body (e.g., one shoulder girdle) or to a single structure at multiple sites (e.g., the peripheral joints) [7].

Once the underlying cause of the disease is diagnosed in rheumatism treatment, the appropriate treatment method is determined. Analgesic and anti-inflammatory drug groups are preferred for rheumatic pain. Due to various side effects, drug interactions and high cost in synthetic drugs, interest in herbal medicines is increasing day by day. Among the population, the variety of plants used against rheumatic pain is quite high.

\section{RESULTS}

In this study, 72 taxa were obtained against various rheumatic pain in various regions of Turkey. Scientific names, families, local names, used parts and usage of these taxa are shown in Table 1.

Table 1. The plants used in traditional treatment against rheumatic pain in Turkey.

\begin{tabular}{|c|c|c|c|c|c|}
\hline Botanical name & Family & Local name & $\begin{array}{l}\text { Plant part } \\
\text { used }\end{array}$ & $\begin{array}{l}\text { Preparation, } \\
\text { administration } \\
\text { and use }\end{array}$ & Ref. \\
\hline Anemone coronaria $\mathrm{L}$. & Ranunculaceae & Lale, Yelotu & Flower & $\begin{array}{l}\text { Ext. to rheumatic } \\
\text { area }\end{array}$ & [8] \\
\hline Apium graveolens L. & Apiaceae & Kereviz & $\begin{array}{l}\text { Seed and } \\
\text { leaf }\end{array}$ & Dec. & [9] \\
\hline $\begin{array}{l}\text { Arctium minus (Hill.) } \\
\text { Bernh. subsp. pubens } \\
\text { (Bab.) Arenes }\end{array}$ & Asteraceae & $\begin{array}{l}\text { Kuncurk, Belg } \\
\text { misek, Acı } \\
\text { kalabak, Kalağan }\end{array}$ & Leaf & $\begin{array}{l}\text { Ext. to rheumatic } \\
\text { area }\end{array}$ & {$[10 ; 11 ; 12]$} \\
\hline Arum elongatum Steven & Araceae & $\begin{array}{l}\text { El kabartan, } \\
\text { Gavur otu, } \\
\text { Kabarcık, } \\
\text { Kabarağı, } \\
\text { Yilancık, Yılan } \\
\text { dili }\end{array}$ & Root & $\begin{array}{l}\text { Ext. to rheumatic } \\
\text { area }\end{array}$ & [13] \\
\hline $\begin{array}{l}\text { Artemisia spicigera } \\
\text { C.Koch }\end{array}$ & Asteraceae & Glyabend & Aerial part & Dec., Int. & [10] \\
\hline Brassica elongata Ehrh & Brassicaceae & $\begin{array}{l}\text { Hardal otu, } \\
\text { Istapan }\end{array}$ & Seed & $\begin{array}{l}\text { Mush, Ext. to } \\
\text { rheumatic area }\end{array}$ & [14] \\
\hline $\begin{array}{l}\text { B. oleracea L. var. acephala } \\
\text { DC. }\end{array}$ & Brassicaceae & Karalahana, Pali & Leaf & $\begin{array}{l}\text { Heated, Ext. to } \\
\text { rheumatic area }\end{array}$ & [5] \\
\hline B. rapa L. var rapa & Brassicaceae & Şalgam & Root & $\begin{array}{l}\text { Grated, Ext. to } \\
\text { rheumatic area }\end{array}$ & [16] \\
\hline Bryonia alba L. & Cucurbitaceae & Yer kabağ1 & Tuber & $\begin{array}{l}\text { Ext. to rheumatic } \\
\text { area }\end{array}$ & {$[11 ; 19]$} \\
\hline $\begin{array}{l}\text { Capparis spinosa L.var. } \\
\text { spinosa }\end{array}$ & Capparaceae & $\begin{array}{l}\text { Gebere, Gevil, } \\
\text { Kapari, Kebere }\end{array}$ & Leaf & $\begin{array}{l}\text { Ext. to rheumatic } \\
\text { area }\end{array}$ & {$[8 ; 18]$} \\
\hline Caucalis platycarpos L. & Apiaceae & $\begin{array}{l}\text { Pitrak, Bitırak, } \\
\text { Düğünotu }\end{array}$ & Aerial part & $\begin{array}{l}\text { Crushed, Ext. to } \\
\text { rheumatic area }\end{array}$ & [19] \\
\hline Cistus laurifolius L. & Cistaceae & $\begin{array}{l}\text { Yavşanak, } \\
\text { Tavşanak, } \\
\text { Tavşancık, } \\
\text { Ladenotu, İldon, } \\
\text { Murt }\end{array}$ & Leaf & $\begin{array}{l}\text { Crushed, Mush } \\
\text { Ext. to rheumatic } \\
\text { area }\end{array}$ & [20] \\
\hline
\end{tabular}


Table 1. (Continued) The plants used in traditional treatment against rheumatic pain in Turkey.

\begin{tabular}{|c|c|c|c|c|c|}
\hline Botanical name & Family & Local name & $\begin{array}{l}\text { Plant part } \\
\text { used }\end{array}$ & $\begin{array}{l}\text { Preparation, } \\
\text { administration } \\
\text { and use }\end{array}$ & Ref. \\
\hline C. salviifolius L. & Cistaceae & $\begin{array}{l}\text { Tavşanak, } \\
\text { Tavşancık }\end{array}$ & Leaf & $\begin{array}{l}\text { Crushed, Mush, } \\
\text { Ext. to rheumatic } \\
\text { area }\end{array}$ & {$[20]$} \\
\hline Clematis flammula L. & Ranunculaceae & Manzaotu, Bahar & Aerial part & $\begin{array}{l}\text { Ext. to rheumatic } \\
\text { area }\end{array}$ & [17] \\
\hline C. vitalba $\mathrm{L}$. & Ranunculaceae & $\begin{array}{l}\text { Karabağ, Deli } \\
\text { asma, Diş otu, } \\
\text { Sarmaşık, Kedi } \\
\text { bağırsağı, } \\
\text { Akbağ, Akçabağ }\end{array}$ & Stem bark & $\begin{array}{l}\text { +Olive oil, Waited, } \\
\text { Ext. to rheumatic } \\
\text { area }\end{array}$ & {$[11]$} \\
\hline $\begin{array}{l}\text { Colchicum speciosum } \\
\text { Steven }\end{array}$ & Liliaceae & $\begin{array}{l}\text { Vargit, Kalkgit, } \\
\text { Çumak, Zumak }\end{array}$ & Seed & $\begin{array}{l}\text { Crushed, Ext. to } \\
\text { rheumatic area }\end{array}$ & {$[21]$} \\
\hline $\begin{array}{l}\text { Cynodon dactylon (L.) } \\
\text { Pers. var. dactylon }\end{array}$ & Poaceae & $\begin{array}{l}\text { Beygit otu, Ayrık } \\
\text { otu, Bıcırgan, } \\
\text { Eklem otu }\end{array}$ & Aerial part & Dec., Int. & [13] \\
\hline $\begin{array}{l}\text { C. dactylon (L.) Pers. } \\
\text { var.villosus Regel }\end{array}$ & Poaceae & Ayrık, Ayrık otu & Rhizome & Dec., Int. & {$[11]$} \\
\hline $\begin{array}{l}\text { Daphne mucronata } \\
\text { Royle }\end{array}$ & Thymelaeaceae & Tevri & Stem bark & $\begin{array}{l}\text { Crushed, Cooked, } \\
\text { Mush, Ext. to } \\
\text { rheumatic area }\end{array}$ & {$[22]$} \\
\hline $\begin{array}{l}\text { Dioscorea communis } \\
\text { (L.)Caddick \& Wilkin }\end{array}$ & Dioscoreaceae & $\begin{array}{l}\text { Dolanbaç, Acı } \\
\text { filiz, Acı ot, } \\
\text { Adem otu, Akıllı } \\
\text { sarmaşık, Köpek } \\
\text { üzümü }\end{array}$ & Root & $\begin{array}{l}\text { Grated, Ext. to } \\
\text { rheumatic area }\end{array}$ & [13] \\
\hline $\begin{array}{l}\text { Diplotaenia cachrydifolia } \\
\text { Boiss. }\end{array}$ & Apiaceae & Siyabu & Root & Dec., Int. & {$[10 ; 22]$} \\
\hline Echium italicum L. & Boraginaceae & $\begin{array}{l}\text { Kuşkonmaz } \\
\text { dikeni }\end{array}$ & Aerial part & $\begin{array}{l}\text { Ext. to rheumatic } \\
\text { area }\end{array}$ & [17] \\
\hline Equisetum arvense L. & Equisetaceae & $\begin{array}{l}\text { Su otu, Kırk } \\
\text { kilitliot, } \\
\text { Minarecik, At } \\
\text { kuyruğu, } \\
\text { Eklemeli ot, } \\
\text { Eklice otu, } \\
\text { Kırkkilit, Kilit } \\
\text { otu, Mide otu }\end{array}$ & Aerial part & $\begin{array}{l}\text { Dec., Ext. to } \\
\text { rheumatic area }\end{array}$ & [13] \\
\hline $\begin{array}{l}\text { Eryngium bithynicum } \\
\text { Boiss. }\end{array}$ & Apiaceae & Boğa dikeni & Aerial part & $\begin{array}{l}\text { Dec., Ext. to } \\
\text { rheumatic area }\end{array}$ & [23] \\
\hline $\begin{array}{l}\text { Eucalyptus } \\
\text { camaldulensis Dehnh. }\end{array}$ & Myrtaceae & $\begin{array}{l}\text { Okaliptus, } \\
\text { Sulfata }\end{array}$ & Leaf & $\begin{array}{l}\text { Essential oil, Ext. } \\
\text { to rheumatic area } \\
\text { Dec., Ext. to } \\
\text { rheumatic area }\end{array}$ & $\begin{array}{l}{[8]} \\
{[24]}\end{array}$ \\
\hline $\begin{array}{l}\text { Fritillaria acmopetala } \\
\text { Boiss. subsp. wendelboi } \\
\text { Pax }\end{array}$ & Liliaceae & Ağlayan gelin & Bulb & $\begin{array}{l}\text { Cooked, Mush, } \\
\text { Ext. to rheumatic } \\
\text { area }\end{array}$ & [25] \\
\hline Galium aparine L. & Rubiaceae & Yapışkan otu & Whole plant & Inf., Int. & [18] \\
\hline Hordeum murinum L. & Poaceae & Kılçık otu, Arpa & Aerial part & $\begin{array}{l}\text { Dec., Ext. to } \\
\text { rheumatic area }\end{array}$ & {$[26]$} \\
\hline
\end{tabular}


Table 1. (Continued) The plants used in traditional treatment against rheumatic pain in Turkey.

\begin{tabular}{|c|c|c|c|c|c|}
\hline Botanical name & Family & Local name & $\begin{array}{l}\text { Plant part } \\
\text { used }\end{array}$ & $\begin{array}{l}\text { Preparation, } \\
\text { administration } \\
\text { and use }\end{array}$ & Ref. \\
\hline $\begin{array}{l}\text { Hypericum perforatum } \\
\text { L. }\end{array}$ & Hypericaceae & $\begin{array}{l}\text { Sarı kantaron, } \\
\text { Kanter çiçeği, } \\
\text { Alaçay, Boyalık } \\
\text { otu, Çayotu, } \\
\text { Kantarot }\end{array}$ & Aerial part & $\begin{array}{l}\text { Crushed in olive } \\
\text { oil, Ext. to } \\
\text { rheumatic area }\end{array}$ & {$[27 ; 28 ; 29]$} \\
\hline Juglans regia $\mathrm{L}$. & Juglandaceae & Ceviz & Fruit & $\begin{array}{l}\text { Dec., Ext. to } \\
\text { rheumatic area } \\
\text { Mush, Ext. to } \\
\text { rheumatic area } \\
\text { Dec., Ext. to } \\
\text { rheumatic area } \\
\text { Ext. to rheumatic } \\
\text { area }\end{array}$ & $\begin{array}{l}{[8]} \\
{[15]} \\
{[19]} \\
{[16 ; 13]}\end{array}$ \\
\hline Laurus nobilis L. & Lauraceae & $\begin{array}{l}\text { Akdeniz defnesi, } \\
\text { Tenel, Tehnel, } \\
\text { Defne }\end{array}$ & $\begin{array}{l}\text { Seed } \\
\text { Oil }\end{array}$ & $\begin{array}{l}\text { Crushed, +Honey, } \\
\text { Eaten } \\
\text { Ext. to rheumatic } \\
\text { area }\end{array}$ & $\begin{array}{l}{[27 ; 30]} \\
{[31]}\end{array}$ \\
\hline $\begin{array}{l}\text { Lavandula officinalis } \\
\text { Chaix }\end{array}$ & Lamiaceae & Lavanta & Aerial part & Inf. & {$[32]$} \\
\hline Linum usitatissimum L. & Linaceae & Keten & Seed & $\begin{array}{l}\text { +Milk, Ext. to } \\
\text { rheumatic area }\end{array}$ & [33] \\
\hline $\begin{array}{l}\text { Matricaria aurea (Loefl.) } \\
\text { Sch.Bip. }\end{array}$ & Asteraceae & $\begin{array}{l}\text { Beybunıc, } \\
\text { Gayeka seva, } \\
\text { Gihake seva, } \\
\text { Çiçeğe zer }\end{array}$ & Aerial part & $\begin{array}{l}\text { Mush, Ext. to } \\
\text { rheumatic area }\end{array}$ & [34] \\
\hline M. chamomilla L. & Asteraceae & $\begin{array}{l}\text { Bopatça, } \\
\text { Papatça, } \\
\text { Papatya, } \\
\text { Keloğlan çiçeği }\end{array}$ & Flower & $\begin{array}{l}\text { Inf., Ext. to } \\
\text { rheumatic area }\end{array}$ & [13] \\
\hline $\begin{array}{l}\text { Medicago rigidula (L.) } \\
\text { All. var. rigidula }\end{array}$ & Fabaceae & Çakırdikeni & Aerial part & $\begin{array}{l}\text { Dec., Int./Mush, } \\
\text { Ext. to rheumatic } \\
\text { area }\end{array}$ & [18] \\
\hline $\begin{array}{l}\text { Mentha longifolia (L.) } \\
\text { Hudson subsp. } \\
\text { longifolia }\end{array}$ & Lamiaceae & $\begin{array}{l}\text { Nane, Su nanesi, } \\
\text { Deli nana, } \\
\text { Yarpuz, Kokulu } \\
\text { nane }\end{array}$ & Leaf & $\begin{array}{l}\text { Mush, Ext. to } \\
\text { rheumatic area }\end{array}$ & [9] \\
\hline $\begin{array}{l}\text { Opuntia ficus- indica } \\
\text { (L.) Miller }\end{array}$ & Cactaceae & $\begin{array}{l}\text { Lap inciri, } \\
\text { Dikenli yemiş, } \\
\text { Eşek dikeni, } \\
\text { Kaynana dili }\end{array}$ & Leaf & Dec., Int. & {$[13]$} \\
\hline Paeonia peregrina Mill. & Paeoniaceae & $\begin{array}{l}\text { Ay gülü, } \\
\text { Beşparmak, } \\
\text { Dolaman, Geyik } \\
\text { lalesi, Top lale }\end{array}$ & Root & $\begin{array}{l}\text { Ext. to rheumatic } \\
\text { area }\end{array}$ & [13] \\
\hline $\begin{array}{l}\text { Pinus nigra Am. subsp. } \\
\text { pallasiana (Lamb.) } \\
\text { Holmboe }\end{array}$ & Pinaceae & Karaçam & Resin & $\begin{array}{l}\text { +Milk, Cooked, } \\
\text { Ext. to rheumatic } \\
\text { area }\end{array}$ & {$[23]$} \\
\hline Pistacia khinjuk Stocks & Anacardiaceae & Gezan, Bittım & Gum & $\begin{array}{l}\text { Ext. to rheumatic } \\
\text { area }\end{array}$ & {$[34]$} \\
\hline
\end{tabular}


Table 1. (Continued) The plants used in traditional treatment against rheumatic pain in Turkey.

\begin{tabular}{|c|c|c|c|c|c|}
\hline Botanical name & Family & Local name & $\begin{array}{l}\text { Plant part } \\
\text { used }\end{array}$ & $\begin{array}{l}\text { Preparation, } \\
\text { administration } \\
\text { and use }\end{array}$ & Ref. \\
\hline $\begin{array}{l}\text { P. terebinthus L. subsp. } \\
\text { terebinthus }\end{array}$ & Anacardiaceae & $\begin{array}{l}\text { Çitlembik, } \\
\text { Çitlemik, } \\
\text { Çetlemik }\end{array}$ & Seed & $\begin{array}{l}\text { Oil, Ext. to } \\
\text { rheumatic area }\end{array}$ & {$[35]$} \\
\hline Platanus orientalis L. & Platanaceae & Çınar & Leaf & $\begin{array}{l}\text { Inf., Int. } \\
\text { Dec., Int. }\end{array}$ & $\begin{array}{l}{[9]} \\
{[13]}\end{array}$ \\
\hline Primula acaulis (L.) L. & Primulaceae & $\begin{array}{l}\text { Zimbon otu, } \\
\text { Menekşe }\end{array}$ & Flower & Crushed & {$[21]$} \\
\hline Prunus spinosa L. & Rosaceae & $\begin{array}{l}\text { Ekşi erik, Dağ } \\
\text { eriği }\end{array}$ & Fruit & Compote, Int. & [36] \\
\hline Ranunculus arvensis L. & Ranunculaceae & $\begin{array}{l}\text { Su pitrağı, } \\
\text { Başdırnağı, } \\
\text { Pıtrak, Sarı } \\
\text { pitrak }\end{array}$ & Aerial part & $\begin{array}{l}\text { Crushed, Ext. to } \\
\text { rheumatic area }\end{array}$ & {$[20]$} \\
\hline $\begin{array}{l}\text { R. constantinopolitanus } \\
\text { (DC.) Dâ'urv. }\end{array}$ & Ranunculaceae & $\begin{array}{l}\text { Çınarcık, } \\
\text { Gağcak, Sakız } \\
\text { otu }\end{array}$ & Leaf & $\begin{array}{l}\text { Mush, Ext. to } \\
\text { rheumatic area }\end{array}$ & [37] \\
\hline R. kotschyi Boiss. & Ranunculaceae & Sarı çiçek & Leaf & $\begin{array}{l}\text { Mush, Ext. to } \\
\text { rheumatic area }\end{array}$ & {$[22]$} \\
\hline R. muricatus L. & Ranunculaceae & Çayır otu & Aerial part & $\begin{array}{l}\text { Crushed, Ext. to } \\
\text { rheumatic area }\end{array}$ & [27] \\
\hline R. pinardii (Stev.) Boiss. & Ranunculaceae & $\begin{array}{l}\text { Gazyağı otu, } \\
\text { Karaz }\end{array}$ & Aerial part & $\begin{array}{l}\text { Crushed, Ext. to } \\
\text { rheumatic area }\end{array}$ & {$[38]$} \\
\hline Raphanus sativus L. & Brassicaceae & $\begin{array}{l}\text { Turp, Yabani } \\
\text { turp otu, Deli } \\
\text { turp otu, } \\
\text { Karaturp }\end{array}$ & Root & $\begin{array}{l}\text { Grated, Ext. to } \\
\text { rheumatic area }\end{array}$ & [39] \\
\hline Rosmarinus officinalis L. & Lamiaceae & Biberiye, kisre & Leaf & $\begin{array}{l}\text { Cooked, Ext. to } \\
\text { rheumatic area }\end{array}$ & {$[40 ; 41]$} \\
\hline Rubus sanctus Schreber & Rosaceae & $\begin{array}{l}\text { Böğürtlen, } \\
\text { Börtlen, Orman } \\
\text { üzümü, Orman } \\
\text { gülü, Kür, } \\
\text { Kocagız kürü }\end{array}$ & Flower & Inf., Int. & [9] \\
\hline Salix alba L. & Salicaceae & Söğüt & Leaf & $\begin{array}{l}\text { Ext. to rheumatic } \\
\text { area }\end{array}$ & [18] \\
\hline S. viminalis L. & Salicaceae & Söğüt & Leaf & Dec., Int. & [39] \\
\hline Sambucus ebulus L. & Caprifoliaceae & $\begin{array}{l}\text { Otsu mürver, Yir } \\
\text { otu, Ayıbogan, } \\
\text { Şahmelek otu, } \\
\text { Piran, Lüver, } \\
\text { Lor, Mürver, } \\
\text { Memer, Sultan } \\
\text { otu, Buzka }\end{array}$ & $\begin{array}{l}\text { Leaf } \\
\text { Root } \\
\text { Aerial part }\end{array}$ & $\begin{array}{l}\text { +Hederae helicis } \\
\text { folium Inf., Ext. to } \\
\text { rheumatic area } \\
\text { Crushed, Ext. to } \\
\text { rheumatic area } \\
\text { Dec., Ext. to } \\
\text { rheumatic area }\end{array}$ & $\begin{array}{l}{[30]} \\
{[37]} \\
{[27 ; 33]}\end{array}$ \\
\hline S. nigra L. & Caprifoliaceae & $\begin{array}{l}\text { Siltan otu, B1zga, } \\
\text { Kokar ot, } \\
\text { Şahmelik, Lüvor, } \\
\text { Melikşah, Deve } \\
\text { kulağ1 }\end{array}$ & $\begin{array}{l}\text { Leafy } \\
\text { branch }\end{array}$ & $\begin{array}{l}\text { Cooked, Ext. to } \\
\text { rheumatic area }\end{array}$ & {$[11]$} \\
\hline
\end{tabular}


Table 1. (Continued) The plants used in traditional treatment against rheumatic pain in Turkey.

\begin{tabular}{|c|c|c|c|c|c|}
\hline Botanical name & Family & Local name & $\begin{array}{l}\text { Plant part } \\
\text { used }\end{array}$ & $\begin{array}{l}\text { Preparation, } \\
\text { administration } \\
\text { and use }\end{array}$ & Ref. \\
\hline Sinapis arvensis L. & Brassicaceae & $\begin{array}{l}\text { Hardal, Sarı } \\
\text { hardal, Kara } \\
\text { hardal }\end{array}$ & $\begin{array}{l}\text { Young } \\
\text { branch }\end{array}$ & $\begin{array}{l}\text { Chopped, Ext. to } \\
\text { rheumatic area }\end{array}$ & [27] \\
\hline Smilax excelsa L. & Smilacaceae & Acıfiliz & Tuber & $\begin{array}{l}\text { Ext. to rheumatic } \\
\text { area }\end{array}$ & {$[42]$} \\
\hline Solanum melongena L. & Solanaceae & Patlican & Fruit stalk & $\begin{array}{l}\text { Cooked in water, } \\
\text { Sediment +Olive } \\
\text { oil, Ext. to } \\
\text { rheumatic area }\end{array}$ & [43] \\
\hline Tamus communis L. & Dioscoreaceae & $\begin{array}{l}\text { Tarla sarmaşı̆̆ı, } \\
\text { Kır sarmaşı̆̆ı, } \\
\text { Çertlemik, } \\
\text { Sincan, Acıot, } \\
\text { Ölüm körüu }\end{array}$ & $\begin{array}{l}\text { Whole plant } \\
\text { Rhizome }\end{array}$ & $\begin{array}{l}\text { Ext. to rheumatic } \\
\text { area } \\
\text { Crushed, Ext. to } \\
\text { rheumatic area }\end{array}$ & $\begin{array}{l}{[44]} \\
{[31]}\end{array}$ \\
\hline $\begin{array}{l}\text { T. communis L. subsp. } \\
\text { cretica (L.) Kit Tan }\end{array}$ & Dioscoreaceae & $\begin{array}{l}\text { Sarmaşık, } \\
\text { Yandıran, Tilki } \\
\text { üzümü }\end{array}$ & $\begin{array}{l}\text { Fruit } \\
\text { Root }\end{array}$ & $\begin{array}{l}\text { Ext. to rheumatic } \\
\text { area } \\
\text { Cutted, Ext. to } \\
\text { rheumatic area }\end{array}$ & $\begin{array}{l}{[11]} \\
{[24]}\end{array}$ \\
\hline $\begin{array}{l}\text { Teucrium chamaedrys L. } \\
\text { subsp. sinuatum(Celak) } \\
\text { Rech.F. }\end{array}$ & Lamiaceae & $\begin{array}{l}\text { Keselmehmut, } \\
\text { Derman }\end{array}$ & Aerial part & Eaten & [22] \\
\hline $\begin{array}{l}\text { Thymbra spicata L. var. } \\
\text { spicata }\end{array}$ & Lamiaceae & $\begin{array}{l}\text { Seyil kekiği, } \\
\text { Kara kekik, } \\
\text { Karaçekme, } \\
\text { Zahter, Bayır } \\
\text { kekiği, Karabaş } \\
\text { otu, Kaya kekiği, } \\
\text { Şeker otu }\end{array}$ & Aerial part & Inf., Int. & [13] \\
\hline $\begin{array}{l}\text { Tilia argentea Desf. ex. } \\
\text { DC. }\end{array}$ & Tiliaceae & Ihlamur & Cortex & $\begin{array}{l}\text { Heated, Ext. to } \\
\text { rheumatic area }\end{array}$ & [9] \\
\hline $\begin{array}{l}\text { Urginea maritima } \\
\text { (L.)Baker }\end{array}$ & Asparagaceae & $\begin{array}{l}\text { Şalgaba, Yakıcı } \\
\text { soğan }\end{array}$ & Bulb & $\begin{array}{l}\text { Core, Ext. to } \\
\text { rheumatic area } \\
\text { Ext. to rheumatic } \\
\text { area }\end{array}$ & $\begin{array}{l}{[24]} \\
{[23]}\end{array}$ \\
\hline Urtica dioica $\mathrm{L}$. & Urticaceae & $\begin{array}{l}\text { Ac1 1sırgan, } \\
\text { Büyük 1sırgan } \\
\text { otu, Gezınk, } \\
\text { C1zlağan, } \\
\text { Dizlağan, } \\
\text { Kopriga }\end{array}$ & $\begin{array}{l}\text { Leaf } \\
\text { Aerial part }\end{array}$ & $\begin{array}{l}\text { Ext. to rheumatic } \\
\text { area/Dec., Ext. to } \\
\text { rheumatic area } \\
\text { Cooked, Mush, } \\
\text { Ext. to rheumatic } \\
\text { area } \\
\text { Ext. to rheumatic } \\
\text { area }\end{array}$ & $\begin{array}{l}{[30 ; 22 ; 15 ;} \\
44 ; 45] \\
\\
{[46]}\end{array}$ \\
\hline U. membranacea Poiret & Urticaceae & $\begin{array}{l}\text { Isirgan, } \\
\text { Karaisirgan, } \\
\text { Dalan }\end{array}$ & Aerial part & $\begin{array}{l}\text { Cooked, Ext. to } \\
\text { rheumatic area } \\
\text { Ext. to rheumatic } \\
\text { area }\end{array}$ & $\begin{array}{l}{[11]} \\
{[8]}\end{array}$ \\
\hline
\end{tabular}


Table 1. (Continued) The plants used in traditional treatment against rheumatic pain in Turkey.

\begin{tabular}{|c|c|c|c|c|c|}
\hline Botanical name & Family & Local name & $\begin{array}{l}\text { Plant part } \\
\text { used }\end{array}$ & $\begin{array}{l}\text { Preparation, } \\
\text { administration } \\
\text { and use }\end{array}$ & Ref. \\
\hline U. urens L. & Urticaceae & $\begin{array}{l}\text { Cizlağan, } \\
\text { Dizlağan, } \\
\text { Isırgan, Isıran }\end{array}$ & $\begin{array}{l}\text { Aerial part } \\
\text { Leaf }\end{array}$ & $\begin{array}{l}\text { Inf., Int. } \\
\text { Crushed, Ext. to } \\
\text { rheumatic area }\end{array}$ & [24] \\
\hline $\begin{array}{l}\text { Verbascum } \\
\text { cheiranthifolium Boiss. } \\
\text { var. cheiranthifolium }\end{array}$ & Scrophulariaceae & $\begin{array}{l}\text { Masicerk, } \\
\text { Bozkulak, Gırç, } \\
\text { Calba, Yalang1 }\end{array}$ & Leaf & $\begin{array}{l}\text { Crushed/ Cooked, } \\
\text { Mush, Ext. to } \\
\text { rheumatic area }\end{array}$ & [10] \\
\hline $\begin{array}{l}\text { V. oreophilum C. Koch } \\
\text { var. joannis (Bords) } \\
\text { Hub.- Mor. }\end{array}$ & Scrophulariaceae & Masicerk & Leaf & $\begin{array}{l}\text { Crushed/ Cooked, } \\
\text { Mush, Ext. to } \\
\text { rheumatic area }\end{array}$ & [10] \\
\hline $\begin{array}{l}\text { V. pyramidatum } \mathrm{M} . \\
\text { Bieb. }\end{array}$ & Scrophulariaceae & Masicerk & Leaf & $\begin{array}{l}\text { Crushed/ Cooked, } \\
\text { Mush, Ext. to } \\
\text { rheumatic area }\end{array}$ & {$[10]$} \\
\hline
\end{tabular}

Int: Internal, Ext: External, Inf: Infusion, Dec: Decoction

Because of its geographical features, Turkey has a very dense plant variety and the use of medicinal plants for the treatment of various diseases among the public since ancient times is widespread. Traditional treatment methods are recorded through ethnobotanical researches and it is aimed to contribute to drug development studies. This study, prepared by screening of ethnobotics researches, revealed 72 taxa that were used against rheumatic pain among the population. These plants are mainly from Ranunculaceae, Asteraceae, Brassicaceae, Lamiaceae, Apiaceae, Liliaceae (Figure 1). Ranunculaceae family contains alkaloids, cardiac and cyanogenetic glycosides; Asteraceae contains volatile oil; Brassicaceae contains mustard-oil glycosides, mucilage and fixed oil; Lamiaceae contains volatile oil; Apiaceae contains volatile oil, coumarins, terpenes and sesguiterpenes, triterpenoid saponins and acetylenic compounds; Liliaceae contains alkaloids, sterols, cardenolides, bufadienolides, steroidal saponins, flavonoids and volatile oil [47]. In these families, some plants contains toxic compounds. But they are usually used externally.

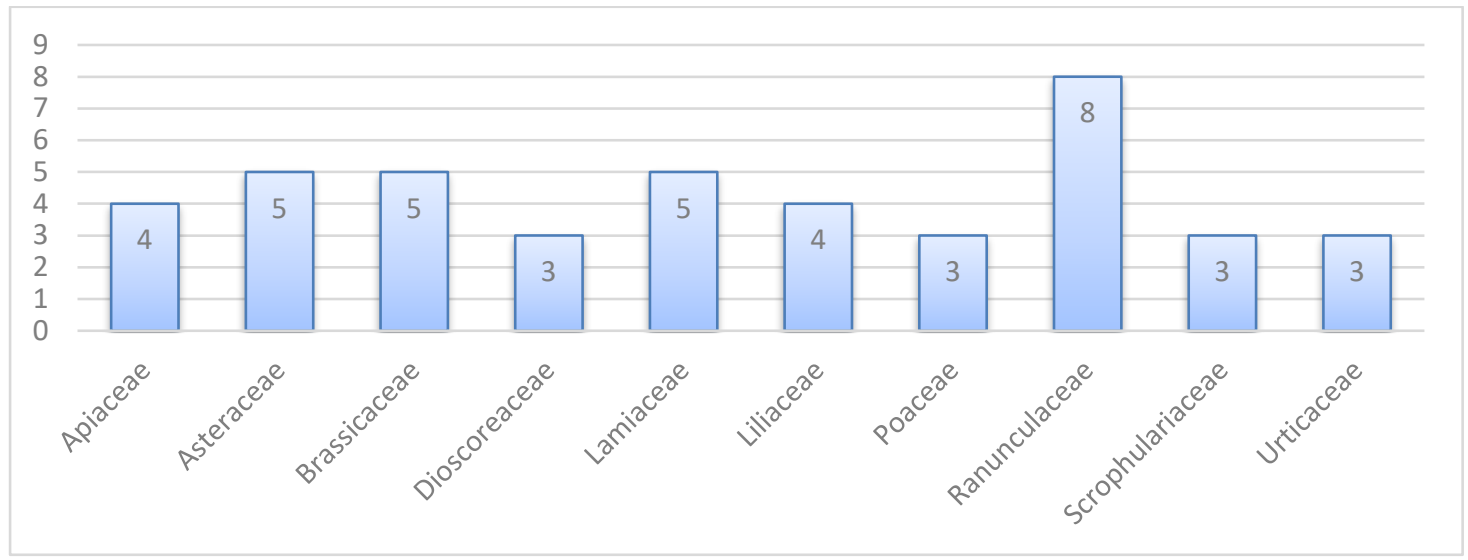

Figure 1. Main families used in traditional treatment against rheumatic pain in Turkey.

Reasons of the usage of plants for the treatment of rheumatoid arthritis is that they contain analgesic, anti-inflammatory, antiseptic, diuretic and rubefacient compounds. For example, the Ranunculaceae family of plants are usually poisonous and contain intensely diverse rubefian substances. Plants containing rubefian compounds (eg Ranunculaceae, Scrophulariaceae, Liliaceae, Dioscoreaceae plants) are applied externally to the rheumatoid area and act by increasing the blood supply of the region. In addition, species exhibiting analgesic, anti-inflammatory (eg Salix alba L.) and antiseptic activity (eg Asteraceae, Lamiaceae, Apiaceae, Urticaceae plants) are also used as decoction / infusion both internally and externally to relieve rheumatic pain. We hope that our study will contribute to new drug development studies that can be used for rheumatic pain. 


\section{MATERIAL AND METHODS}

This study is prepared by searching MSc and PhD theses at the National Higher Education Center and ethnobotanical surveys conducted in various parts of the Turkey with selecting regional plants used for rheumatic pain.

Author contributions: Concept - M.Ş.E., S.A., G.M.; Design - M.Ş.E., S.A., G.M.; Supervision - M.Ş.E., S.A., G.M.; Resource - M.Ş.E., S.A., G.M.; Materials - M.Ş.E., S.A., G.M.; Data Collection and/or Processing M.Ş.E., S.A., G.M.; Analysis and/or Interpretation - M.Ş.E., S.A., G.M.; Literature Search - M.Ş.E., S.A., G.M.; Writing - M.Ş.E., S.A., G.M.; Critical Reviews - M.Ş.E., S.A., G.M.

Conflict of interest statement: The authors declared no conflict of interest.

\section{REFERENCES}

[1] Yüceer S. MSc Thesis. Knowledge of nursing students related fifth vital sign pain and postoperative pain. Nursing Department, Faculty of Health Sciences, Hacettepe University, Ankara, 2008.

[2] Erdine S. İstanbul Ağr1 Merkezi. Available in: www.agritr.com/html/algoloji.html [Accessed: 19.03.2017].

[3] Güner A, Özhatay N, Ekim T, Başer KHC. Flora of Turkey and the East Islands, vol. 11. Edinburgh University Press, Edinburgh 2000.

[4] Özhatay N, Kültür Ş, Gürdal B. Check-list of Additional Taxa to the Supplement Flora of Turkey VI. J Fac Pharm Istanbul 2013; 43 (1): 33-82.

[5] Özhatay N, Kültür Ş, Gürdal B. Check-list of Additional Taxa to the Supplement Flora of Turkey VII. J Fac Pharm Istanbul 2015; 45 (1): 61-86.

[6] Engin B. Romatizma: Nedenleri, belirtileri, tanısı ve tedavisi. Available at: http://www.xn--salk1wa3i.net/romatizma.html [Accessed: 19.03.2017].

[7] Hardin JG. Clinical Methods: The History, Physical and Laboratory Examinations. Chapter 159: Rheumatic Pain. Available in: https://www.ncbi.nlm.nih.gov/books/NBK267/ [Accessed: 19.03.2017].

[8] Gürdal B, Kültür Ş. An ethnobotanical study of medicinal plants in Marmaris (Muğla, Turkey). J Ethnopharmacol. 2013; 146: 113-126.

[9] Sargin SA, Akçiçek E, Selvi S. An ethnobotanical study of medicinal plants used by the local people of Alaşehir (Manisa) in Turkey. J Ethnopharmacol. 2013; 150(3): 860-874.

[10] Mükemre M, Behçet L, Çakılcığlu U. Ethnobotanical study on medicinal plants in villages of Çatak (Van Turkey). J Ethnopharmacol 2015; 166: 361-374.

[11] Aktan T. MSc Thesis. The Ethnobotanical examine in the villages of Yenişehir (Bursa). Department of Biology, Celal Bayar University, Manisa, 2011.

[12] Özgen U, Coşkun M. Ilıca (Erzurum) ilçesine bağlı köylerde halk ilacı olarak kullanılan bitkiler. 13. Bitkisel İlaç Hammaddeleri Toplantısı (İstanbul, 20-22 Eylül 2000) “Bildiri Kitabı” Gürkan E, Tuzlacı E (Eds.). MÜ Ecz. Fak. Yay. No: 17. İstanbul. 2001, pp.135-143.

[13] Özdemir Nath E. PhD Thesis. An ethnobotanical study in Savaştepe and Kepsut region (Balıkesir). Department of Pharmaceutical Botany, Institue of Health Science, Istanbul University, 2016.

[14] Ezer N, Avcı K. Çerkeş (Çankırı) Yöresinde kullanılan halk ilaçları. Hacettepe Univ J Fac Pharm. 2004; $24(2)$ : 67-80.

[15] Kızılarslan Ç, Özhatay N. Wild plants used as medicinal purpose in the south part of İzmit (Northwest Turkey). Turk J Pharm Sci. 2012; 9(2): 199-218.

[16] Sadıkoğlu N, Alpınar K. Etnobotanik açıdan Bartın. 13. Bitkisel İlaç Hammaddeleri Toplantısı (İstanbul, 20-22 Eylül 2000) “Bildiri Kitabı” Gürkan E, Tuzlacı E (Eds.). M.Ü.Ecz. Fak. Yay. No: 17. İstanbul. 2001, pp.87.

[17] Yeşilada E, Honda G, Sezik E, Tabata M, Fujita T, Tanaka T, Takeda Y, Takaishi Y. Traditional medicine in Turkey. V. Folk medicine in inner Taurus Mountains. J Ethnopharmacol. 1995; 46: 133-152.

[18] Özhatay N, Koçak S. Plants used for medicinal purposes in Karaman province (Southern Turkey). İstanbul Ecz Fak Derg. 2010-2011; 41.

[19] Şenkardeş İ. PhD Thesis. Ethnobotanical investigations in southern districts (Acıgöl, Derinkuyu, Gülşehir, NevşehirCentral district, Ürgüp) of Nevşehir. Department of Pharmaceutical Botany, Marmara University, Istanbul, 2014.

[20] Kargığlu M, Cenkci S, Serteser A, Konuk M, Vural G. Traditional uses of wild plants in the middle Aegean region of Turkey. Hum Ecol. 2010; 38: 429-450. 
[21] Akbulut S, Ozkan ZC. Traditional usage of some wild plants in Trabzon region (Turkey), Kastamonu Univ. J Forestry Fac. 2014; 14(1): 135-145.

[22] Kaval İ, Behçet L, Cakilcioglu U. Ethnobotanical study on medicinal plants in Geçitli and its surrounding (Hakkari - Turkey). J Ethnopharmacol. 2014; 155(1): 171-184.

[23] Sezik E, Yeşilada E, Honda G, Takaishi Y, Takeda Y, Tanaka T. Traditional medicine in Turkey X. Folk medicine in Central Anatolia. J Ethnopharmacol. 2001; 75 (2-3): 95-115.

[24] Tuzlacı E. Şifa Niyetine Türkiye' nin Bitkisel Halk İlaçları, Alfa Basım Yayım Dağıtım Ltd. Şti., 1. Basım 2006.

[25] Erdoğan R. MSc Thesis. Ethnobotanical features some of the wild on the Sarıveliler (Karaman) and its environment ethnobotanic. Department of Biology, Selçuk University, Konya, 2011.

[26] Doğru Koca A, Yıldırımlı Ş. Ethnobotanical properties of Akçakoca district in Düzce (Turkey). Hacettepe J Biol Chem. 2010; 38(1): 63- 69.

[27] Ecevit Genç G, Özhatay N. An Ethnobotanical Study in Çatalca (European Part of Istanbul) II. Turk J Pharm Sci. 2006; 3(2): 73-89.

[28] Özgökçe F, Özçelik H. Ethnobotanical aspects of some taxa in East Anatolia, Turkey. Econ Bot 2004; 58 (7): $697-704$.

[29] Polat R. PhD Thesis. Agricultural biodiversity and ethnobotanical research in the Havran and Burhaniye regions of Balıkesir. Department of Biology, Balıkesir University, Balıkesir, 2010.

[30] Saraç DU, Özkan ZC, Akbulut S. Ethnobotanic features of Rize/Turkey province. Biol Divers Conserv. 2013; 6(3): 57-66.

[31] Duran A. Akseki (Antalya) ilçesindeki bazı bitkilerin yerel adları ve etnobotanik özellikleri. OT Sistematik Botanik Derg. 1998; 5(1): 77-92.

[32] Şahin YiğitS. MSc Thesis. Medicinal plants sold in Gaziantep herbalists and their ethnobotanical aspects. Department of Biology, Gaziantep University, Gaziantep, 2014.

[33] Yeşilada E, Sezik E, Honda G, Takaishi Y, Takeda Y, Tanaka T. Traditional medicine in Turkey IX: Folk medicine in north- west Anatolia. J Ethnopharmacol. 1999; 64: 195-210.

[34] Akan H, Korkut MM, Balos MM. Arat Dağı ve çevresinde (Birecik, Şanlıurfa) etnobotanik bir araştırma. Fırat Üni Fen Müh Bil Derg. 2008; 20 (1): 67-81.

[35] Alkaç SA. MSc Thesis. Alaçam mountains (Balıkesir) from Bigadiç city area around economic importance of some plants and features ethnobotany. Department of Biology, Balıkesir University, Balıkesir, 2013.

[36] Türkan Ş, Malyer H, Özaydın S, Tümen G. Ordu ili ve çevresinde yetişen bazı bitkilerin etnobotanik özellikleri. Süleyman Demirel Üni Derg 2006; 10(2): 162-166.

[37] Demirci S, Özhatay N. An ethnobotanical study in Kahramanmaraş (Turkey); Wild plants used for medicinal purpose in Andırın, Kahramanmaraş. Turk J Pharm. Sci. 2012; 9(1): 75-92.

[38] Yeşil Y, Akalın E. Folk medicinal plants in Kürecik area (Akçadağ/Malatya - Turkey). Turk J Pharm Sci. 2009; 3: 207220.

[39] Metin A. Msc Thesis. Ethnobotanical features of plants in Mut (Mersin) and its environments. Department of Biology, Selçuk University, Konya, 2009.

[40] Saday H. MSc Thesis. Ethnobotanical properties of Güzeloluk village and its vicinity. Department of Biology, Selçuk University, Konya, 2009.

[41] Eşen B. MSc Thesis. The ethnobotanical properties of Aydınlarvillage and its vicinity (Erdemli/ Mersin). Department of Biology, Selçuk University, Konya, 2008.

[42] Saçlı S, Akalın E. Preliminary ethnobotanical study from Kaz dağı (Balıkesir/ Çanakkale) I: Uses and vernacular names. J Fac Pharm Istanbul 2001; 34(2): 9-16.

[43] Güneş S, Savran A, Paksoy MY, Koşar M, Çakılcığlu U. Ethnopharmacological survey of medicinal plants in Karaisalı and its surrounding (Adana-Turkey). J Herb Med; 2017.

[44] Poyraz Kayabaşı N. MSc Thesis. The ethnobotanical examine in the villages of Manyas and Manyas. Department of Biology, Balıkesir University, Balıkesir, 2011.

[45] Keskin L. MSc Thesis. Kadınhanı (Konya) ve çevresinde yetişen bitkilerin etnobotanik özellikleri. Department of Biology, Selçuk University, Konya, 2011.

[46] Kültür Ş. Medicinal plants used in Kırklareli province (Turkey). J Ethnopharmacol. 2007; 111: 341-364.

[47] Baytop T. Türkiye'de Bitkiler ile Tedavi, Geçmişte ve Bugün. Nobel Medicine Publication, İstanbul. 1999. 